\title{
ENSEÑANZA DEL USO DE LAS TIC A PERSONAS MAYORES: RELATO DE UNA EXPERIENCIA
}

\section{Teaching the use of ICT to older people: an account of an experience}

\author{
Cristina Moreno Pabón \\ Facultad de Educación. Universidad Autónoma de Madrid \\ Correo-e: cristina.moreno@uam.es \\ Cristina Alconada Fernández \\ Experta en Tecnología Educativa, profesora de Pluma y Arroba \\ Correo-e: c.alconada@gmail.com \\ José Dulac Ibergallartu \\ Director de Pluma y Arroba y de la investigación Aula Adaptativa \\ Correo-e: jose@dulac.es
}

Recepción: Io de diciembre de 2017

Envío a informantes: is de diciembre de 2017

Aceptación definitiva: 20 de enero de 2018

Resumen: En este artículo presentamos la investigación que desarrollamos desde hace dos años trabajando con recursos y herramientas TIC para enseñar a las personas mayores su uso, funcionalidades y beneficios que les pueden aportar. Consideramos que en la sociedad actual no hemos dado una respuesta satisfactoria a la formación de este colectivo de personas mayores que pensamos que está desfavorecido y lo estamos considerando una necesidad ante los constantes avances y cambios referidos a las tecnologías. Las herramientas que hemos incorporado son la pizarra digital y tabletas para navegación por internet y uso de algunas aplicaciones relacionadas con los dispositivos Android y referidas al dibujo y las mejoras cognitivas; robots básicos para iniciar la programación, la implicación emocional y el pensamiento lógico, y gafas de realidad virtual para acercar la novedad inmersiva en el conocimiento o recuerdo de diferentes entornos. Los buenos resultados obtenidos en este periodo nos animan a continuar y consolidar el proyecto de investigación y definir los modelos didácticos más adecuados y las competencias básicas digitales pertinentes.

Palabras Clave: Tecnología Educativa; pizarra digital; personas mayores; TIC. 
АвsтRAст: In this article, we present the research we have been developing for the last two years, working with ICT resources and tools to teach older people about its use, functionalities and benefits that can be provided to them. We believe that in today's society we have not given a satisfactory response to the formation of this group of older people that we think is disadvantaged in terms of the use of ICT training and we are considering it a necessity in the face of constant advances and changes related to technologies. The tools we have incorporated are the digital board and tablets for Internet browsing and use of some applications related to Android devices and referred to drawing and cognitive improvements, basic robots to start programming, emotional involvement and logical thinking and glasses of virtual reality to bring the immersive novelty in the knowledge or memory of different environments. The good results obtained in this period encourage us to continue and consolidate the research project and define the most appropriate didactic models and the relevant digital basic competences.

KeY words: Educational Technology; Digital Whiteboard; Seniors; ICT.

\section{Antecedentes}

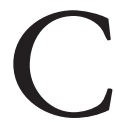

ON LA INCORPORACIÓN DE LAS PIZARRAS DigitAles EN LAS AUlas en el año 2004 descubrimos un nuevo potencial de comunicación y colaboración en el que merecía la pena iniciar una investigación para detectar las mejoras en la enseñanza y definir los modelos didácticos más adecuados ante la cada vez mayor digitalización en los procesos de enseñanza y aprendizaje. De las aulas ordinarias de colegios, centros de formación y universidades fuimos participando en diferentes estudios para incorporar la herramienta en otros ámbitos en los que mejoraba la comunicación y la colaboración entre los participantes. Empresas, sanidad, ejército... y decidimos acercarnos al mundo de las personas mayores que están en residencias de ancianos.

Cuando iniciamos la construcción de un marco teórico nos encontramos con pocos trabajos que relacionasen las TIC con los ancianos y, sobre todo, pocos que se dilatasen en un periodo de tiempo más o menos largo para consolidar los estudios realizados. Es difícil conseguir proyectos que perduren. Nosotros consideraríamos un plazo superior a los doce meses, dadas las características de los participantes en el proyecto, para consolidar los aprendizajes y mejoras obtenidos. Es una suerte comenzar nuestro tercer año de proyecto con el mismo grupo de mayores.

Todos los estudios e investigaciones coincidían en sus informes en los beneficios aportados cuando las personas mayores utilizaban las TIC, pero no encontramos ningún proyecto que consolidase una enseñanza continuada en el tiempo, estructurada como adquisición de competencias digitales básicas adaptadas e integrada en las rutinas de las actividades programadas en terapia ocupacional, así que esto nos animó a desarrollar el proyecto actual.

Dos metodologías que aplicábamos en nuestras aulas y sobre las que realizamos investigaciones consolidadas nos sirvieron de base para la implementación y mejora del proyecto. Aula Adaptativa con sus Laboratorios y MeTaEducArte. 
ENSEÑANZA DEL USO DE LAS TIC A PERSONAS MAYORES:

RELATO DE UNA EXPERIENCIA

C. MORENO PABÓN, C. ALCONADA FERNÁNDEZ Y J. DULAC IBERGALLARTU

\section{Justificación}

Consideramos que el sector de las personas mayores es uno de los más necesitados en una justicia social ante los constantes cambios que se producen y que les alejan de la realidad y vivencias del mundo de hoy. La población mundial y la española prosiguen un envejecimiento continuo. Los datos del Informe sobre personas mayores en España del Consejo Superior de Investigaciones Científicas (CsIC) nos indican que España sigue su proceso de envejecimiento. Según los datos del Padrón Continuo (INE) a I de enero de 2015 hay 8.573.985 personas mayores (65 y más años), el I8,4\% sobre el total de la población, 46.624.382 (Abellán y Pujol, 2016: 3).

Parece necesario, teniendo en cuenta los cambios y avances tecnológicos y el aumento de la población de mayores, dedicar una especial atención educativa a este colectivo al que todos pensamos llegar a pertenecer. Sabemos que no es una necesidad suficientemente cubierta en los ámbitos de convivencia de los ancianos y queremos aportar nuestra experiencia con la investigación y el proyecto.

\section{El proyecto}

El presente proyecto se inicia en el mes de noviembre de 20I5. Llevábamos trabajando más de diez años con pizarras digitales en las aulas y las investigaciones desarrolladas nos descubrían un apasionante nuevo modo de comunicar en el aula con mutimedia de calidad añadiendo interactividad y colaboración que permitían definir nuevos modelos didácticos. Desde hacía tiempo deseábamos llevar la pizarra digital, la herramienta mejor y más novedosa con la que trabajábamos en las aulas, a una residencia de ancianos para que ellos la pudieran utilizar y descubrir algunas de sus posibilidades.

Ya en el año 2005, la investigación sobre el uso de la pizarra digital en las aulas denominada Iberian Reseach Proyect detectaba los beneficios de la herramienta como ayuda para la mejora de la enseñanza y afirmaba en sus conclusiones:

- La Pizarra Digital mejora notablemente los procesos de enseñanza y aprendizaje.

- La Pizarra Digital es una herramienta tecnológica muy bien aceptada por los profesores.

- La utilización de la Pizarra Digital en las aulas motiva a alumnos y profesores. Gallego y Dulac (2005).

Hemos tenido que ir haciendo constantes adaptaciones y seguimos adaptándonos ya que el tiempo nos ha ido enseñando a todos, desde las primeras sesiones cercanas a la Navidad en las que dibujamos en la pizarra digital, después en tabletas, felicitaciones navideñas, hasta las últimas sesiones de noviembre de 2017 en las que programamos con robots. La evolución del proyecto ha aplicado en todo momento el método adaptativo de nuestra investigación Aula Adaptativa. Debíamos adaptar y reinventar los talleres, a veces sobre la marcha, para poder conseguir los objetivos que nos habíamos propuesto. Los aspectos emocionales y de relación entre los alumnos se trabajaron con el método MeTaEducArte. Trataremos esto con más detalle en el epígrafe Metodología. 
Así, el proyecto ha ido tomando forma y se ha consolidado. Comenzamos el tercer año y eso nos da una consistencia de tiempo, método y resultados que afianza nuestra investigación.

Hemos decidido realizar cuatro talleres que son los más adecuados para conseguir una visión global del uso de las Tic en nuestro perfil de alumnos, para nuestra investigación y en los que también estamos trabajando en las aulas ordinarias.

- Taller de pizarra digital. Felicitaciones navideñas. Internet y presentaciones.

- Taller de tabletas. Fotografía. Dibujo. Aplicaciones. Internet.

- Taller de Internet. Búsqueda de información, de lugares recordados en los que habían vivido, ciudades, canciones, películas, personajes...

- Taller de robótica. Para conocer algunos de los robots más populares e iniciarse en la programación. Destacamos el vínculo con robots que emocionan y motivan.

- Taller de realidad virtual. Para acceder y conocer mundos virtuales, entornos y lugares con el potencial de la inmersión.

\section{Objetivos}

Nuestro objetivo inicial era de manera sencilla y casi anecdótica el acercar la tecnología de la pizarra digital a las personas mayores. Aprovechamos para realizar felicitaciones navideñas. A partir de la primera experiencia hemos ido definiendo los objetivos de la investigación.

- Acercar las TIC a las personas mayores bajo un entorno motivador, fácil, amigable, eficaz y colaborativo.

- Decidir las tecnologías más adecuadas para ello. Herramientas y recursos.

- Adaptar las metodologías utilizadas en nuestras investigaciones al entorno de personas mayores.

- Aportar datos a las investigaciones sobre estilos de aprendizaje.

- Detectar algunas de las mejoras de los mayores participantes en el proyecto en cuanto a capacidades cognitivas, emocionales, de relaciones sociales personales y de la autoestima.

\section{Recursos}

Una característica importante del proyecto ha sido que lo estamos llevando a cabo con unos mínimos recursos económicos. No así humanos, que son los más importantes y en los que hemos contado con importantes ayudas. Hasta el momento en el que escribimos este artículo, no hemos recibido ningún tipo de ayuda económica. Nuestro primer reto fue conseguir una pizarra digital e instalarla en una residencia para iniciar unos talleres que en principio era nuestro primer y único objetivo.

Nos dirigimos a la Residencia Orpea Buenavista (Madrid), en la que tener un familiar residente nos posibilitó tanto el acceso como el conocer las dinámicas con las que trabajan y que se adecúan al proyecto que queríamos iniciar. Los residentes y sus 
ENSEÑANZA DEL USO DE LAS TIC A PERSONAS MAYORES:

RELATO DE UNA EXPERIENCIA

actividades y rutinas están organizados en función de su autonomía y de sus capacidades cognitivas. El grupo con el que trabajamos no presentan deterioros cognitivos o tienen deterioros leves o moderados y son funcionalmente suficientes. Desde un principio contamos con la colaboración de la psicóloga del centro que se ocupó de proponer a los residentes más idóneos la participación en este proyecto. También contamos con su asesoramiento en cuanto a las dinámicas más adecuadas: duración de las sesiones, agrupamientos...

En la primera fase del proyecto se desarrolló en una sala amplia, multiusos, en la que se trabajó con una pizarra digital dibujando felicitaciones de Navidad, que fue la primera actividad que se realizó. Dos años después repetimos la experiencia y pudimos disfrutar de las mejoras y avances de nuestros alumnos mayores trabajando con las aplicaciones de las tabletas.

Posteriormente, al ampliar la variedad de herramientas utilizadas, pasamos al aula de terapia ocupacional. Allí trabajamos tanto en grupos de tres o cuatro alumnos como en gran grupo. El mobiliario permite la distribución más adecuada a cada situación, sin que las sillas de ruedas, andadores y muletas, tan frecuentes, supongan un impedimento. Los recursos humanos han sido lo más importante del proyecto, comenzando por nuestros alumnos. Unos treinta hemos tenido a lo largo de estas ediciones, afortunadamente ha habido muy pocas bajas y ninguna deserción, algo inhabitual en este perfil de alumnos.

El absentismo tampoco ha sido un factor determinante negativo, todo lo contrario, ya que podríamos considerarlo en torno al $15 \%$. No siempre asisten todos, enfermedades, visitas de familiares, olvidos..., y cuando esto sucede nos suelen pedir disculpas en la sesión siguiente, lamentándose tanto por «el feo»-dicen ellos-que nos hacen a nosotros, como por lo que no han podido aprender y esto sí que nos parece importante, el haberles despertado la seguridad de que pueden seguir aprendiendo y el interés por ello. Como ya se ha dicho más arriba, estos alumnos o no tienen deterioro cognitivo o es muy leve. Sus edades oscilan entre los 78 y los 97 años, no siendo los más ancianos los menos lúcidos ni menos entusiastas. Fuera de este rango de edad hay dos alumnos, en la sesentena, con otro tipo de problemática a pesar de la edad. Todos ellos participan voluntariamente y esperan las sesiones con verdadero interés. A muchos les ha abierto una ventana al mundo exterior que creían cerrada para siempre: acceso a la información, poder participar más activamente en las conversaciones familiares, poder compartir con sus compañeros vivencias que solo la imagen puede transmitir con la exactitud que ellos quieren, sobre todo los que han vivido en el extranjero y les pueden explicar a los demás cómo era allí su vida, son algunos de los ejemplos de situaciones reales de cada sesión. Y no digamos volver a oír canciones de las que no recuerdan más que una frase o verse programando un robot muy básico, pero robot al fin y al cabo, que ejecuta las acciones que ellos han diseñado.

El personal de psicólogos de la residencia colaboran con entusiasmo y asesoran desde el mismo momento en que les planteamos el proyecto, dejándonos, por otra parte, autonomía para decidir el tipo de actividades y las herramientas y recursos a utilizar. Periódicamente mantenemos reuniones para ir analizando el camino recorrido, los logros conseguidos, los giros y adaptaciones que siempre conviene hacer para ir adecuándose a las circunstancias variables y buscar mejoras.

Este curso se ha incorporado el equipo de terapeutas. En la reunión que mantuvimos a principio de curso con la directora y la psicóloga convinimos en que el 
proyecto estaba lo suficientemente consolidado como para integrarlo en las rutinas y actividades periódicas, por lo que, además de dejar allí estable el material que llevábamos para cada sesión, tabletas y ordenador táctil fundamentalmente, empezamos a coordinarnos con los terapeutas ocupacionales que han sistematizado las actividades a realizar con estas herramientas, mientras que cuando vamos nosotros aportamos otras como robots o gafas de realidad virtual, otras modalidades del uso de internet y las aplicaciones ampliando así esa aplicación de la tecnología en sus actividades.

El equipo de profesores participante en el proyecto está formado por:

Cristina Moreno Pabón, profesora de la Facultad de Educación de la Universidad Autónoma de Madrid. Creadora del Proyecto y método MeTaEducArte; Cristina Alconada Fernández, profesora investigadora y formadora en Tecnología Educativa; Dulac Ibergallartu, profesor, director de Pluma y Arroba y de la investigación Aula Adaptativa, y Claudia Uzkuras, profesora de educación secundaria y miembro de la investigaciòn Aula Adaptativa.

Es un proyecto que «engancha», dicho en términos coloquiales, y son varias las personas que se han ofrecido a acompañarnos en estas sesiones. Lo difícil es coordinar los días y horarios de talleres con el equipo docente. Son situaciones enriquecedoras que le obligan a uno a sacar lo mejor de uno mismo, como persona y como docente, porque si importante es la capacidad de empatizar con dificultades como el temblor del pulso al pulsar un icono no lo es menos buscar la forma de expresión adecuada para explicar un proceso que nosotros damos por sabido de puro repetido.

Partimos de unos medios materiales básicos. Instalamos una pizarra digital de 80 pulgadas y llevábamos nuestros proyector y ordenador a cada unos de los talleres. Nuestros alumnos necesitaban una atención muy personalizada y una única herramienta nos resultaba insuficiente tras las primeras dos sesiones y optamos por llevar nuestras tabletas digitales y teléfonos, con lo que ya podíamos hacer por grupos reducidos la misma actividad que realizábamos en la pizarra. Hemos tenido que ir aumentando esta dotación tecnológica inicial según el proyecto ha ido avanzando hasta disponer de un material para los talleres consistente en: una pizarra digital, dos ordenadores, un proyector, 8 tabletas 4 robots y cuatro gafas de realidad virtual.

Los softwares y las aplicaciones que se están utilizando son cuidadosamente seleccionados para conseguir un uso rentable debiendo ser fáciles y motivadores, demostrando un resultado muy rápido en el avance del aprendizaje de nuestros mayores. Por supuesto que navegar por internet y mejor en la pizarra digital ha sido el recurso de mayor impacto.

Algunas de las aplicaciones y softwares utilizados han sido:

- Chrome Navegador de internet, que integramos muy bien con los recursos en Drive.

- Mapas de Google. Conocemos, recordamos, visitamos lugares.

- Paint Joy. Sencillo software para dibujar con unos colores luminosos.

- Memory. Para recordar y descubrir parejas.

- Sopa letras. Fáciles y de un tamaño adecuado. De dificultad creciente.

- Rompecabezas. Permite realizar sencillos puzles.

- Robot Doc. Inicia en los conceptos de programación básica.

- Cardboard. Nos permite iniciarnos en la realidad virtual. 
ENSEÑANZA DEL USO DE LAS TIC A PERSONAS MAYORES:

RELATO DE UNA EXPERIENCIA

C. MORENO PABÓN, C. ALCONADA FERNÁNDEZ Y J. DULAC IBERGALLARTU

\section{Metodología}

Lo que nació como un taller se fue convirtiendo en todo un proyecto en el que nos íbamos enredando y motivando al observar los buenos resultados. Nuestros mayores mejoraban notablemente en su autoestima y procesos cognitivos y deseaban conocer más. Se sentían capaces de ello como manifiestan con frecuencia: «Lo que más me gusta es saber que yo también soy capaz de utilizar estas tecnologías».

Ya desde las primeras reuniones y talleres tuvimos que aprender a enseñar a nuestros nuevos alumnos con métodos muy diferentes de los que estábamos habituados. Uno de los primeros aspectos que detectamos fue la necesidad de priorizar, mucho más que en nuestras aulas ordinarias, la competencia emocional; porque en educación es imposible que se dé el correcto proceso enseñanza-aprendizaje si no hay emoción, pero en este caso, con estos alumnos, el concepto se revisa, se renueva y se antepone a todo lo demás. Estamos ante unos alumnos que han tenido ya un recorrido vital dilatado, algunos con un bagaje francamente interesante; otros, más plano, pero todos comparten la sensación de haber llegado al final. Al final de lo que podían conocer, de lo que podían aprender, de lo que podían compartir y hasta enseñar. Por todo esto es muy importante poner especial cuidado en la forma de relacionarnos con ellos y creemos que el concepto que mejor lo resume es del «respeto valorativo», ni conmiseración, ni impaciencia, ni ese proteccionismo que les despoja de su dignidad y que tanto les desagrada. No son como niños y no hay que tratarlos como tales. Hay que escuchar, pero no con paciencia, sino con interés. Hay que mirarles a los ojos cuando hablan, porque para muchos de ellos ha pasado mucho tiempo desde la última vez que se les escuchó con atención, de igual a igual. Y hay que valorar positivamente lo que aportan porque a partir de estas experiencias iremos elaborando los contenidos en presentación multimedia e interactivos basados en sus centros de interés, como se irá viendo a lo largo de este artículo.

En un principio pensamos que un profesor podría atender a ocho o diez alumnos, con lo que con dos profesores, tres a lo sumo, eran suficientes para un aula de veinte alumnos que venía a ser la media de asistencia en un principio entre olvidos, enfermedades y visitas de parientes. Pero pronto nos dimos cuenta de que es necesario un equipo mayor; tanto porque las ausencias son cada vez menos frecuentes -a veces, los hijos que van a verles nos piden estar en la sesión porque los padres prefieren quedarse o les tienen esperando fuera-, como porque el tipo de actividades y su actitud que demanda atención hacen idónea una ratio de un profesor, como máximo, cada cuatro alumnos, sobre todo hasta que desarrollan la suficiente autonomía ante la nueva herramienta, aplicación o actividad. Y porque, y esta es una característica muy destacada en este tipo de alumnos, les cuesta estar ociosos esperando a que sus compañeros acaben para hacer o intervenir ellos. Les cuesta esperar y escuchar a los demás, salvo en las presentaciones de sus lugares y centros de interés, por ejemplo, donde saben que como ellos escuchan serán escuchados después.

Muchos alumnos tienen problemas a la hora de sincronizar los movimientos, les tiembla el pulso; otros no ven bien, y la mayoría oye mal. Todo un reto cuando se quiere animar a utilizar herramientas que no están diseñadas para suplir estas carencias. No porque no las haya, sino porque los recursos económicos solo nos permiten utilizar los que están en el mercado habitual, aun así lo importante es transmitirles la suerte que tenemos de disponer de estos recursos. ¿Qué hacemos? En primer lugar, 
no utilizar pantallas menores de 9 pulgadas, ampliar todo lo posible el tamaño de los iconos, marcar con gomets adhesivos los botones de encendido y apagado... y, sobre todo, tener la confianza de que personas que a lo largo de su vida han ido superando dificultades también pueden hacer frente a estas carencias. Y esto también es educativo porque les supone un reto que les resulta estimulante. El poder utilizar los mismos aparatos, como dicen ellos, que sus hijos y sus nietos les fomenta mucho la autoestima con todos los beneficios que ello reporta a todos los niveles. Resulta gracioso oírles despreciar el teléfono móvil para tontos (como ellos dicen) que tienen, básico y sencillo. Pensando que serían capaces de utilizar teléfonos inteligentes, aunque en realidad hemos comprobado que las pantallas de 7 pulgadas y las de los móviles les resultan muy difíciles de gestionar por lo indicado anteriormente.

Necesitábamos implementar una metodología y adaptarla a esta nueva forma de enseñar y de aprender en la que comenzábamos a investigar. Para ello nos propusimos conocer y definir cómo son los estilos de aprendizaje de las personas mayores. Desde que en 2003 conocimos de la mano de Domingo Gallego y Catalina Alonso -que fue quien recogió y adaptó el cuestionario Honey de Estilos de Aprendizaje al entorno y al idioma español, creando el CHAEA-, lo hemos venido aplicando en diferentes situaciones formativas, tanto presenciales como online. Esta dilatada experiencia, junto con la actitud receptiva y entusiasta ante todo lo que se les propone, nos indujo a plantearnos el trabajarlo con nuestros alumnos mayores.

En un principio les aplicamos el cuestionario de I2 ítems adaptado por Antonio Fernandes, de Portugal. Se les aplicó a 23 alumnos, con edades comprendidas entre los 80 y los 92 años. En este proceso conviene destacar dos circunstancias: la aplicación de la prueba y los resultados.

En cuanto a la aplicación de la prueba, hay que reseñar la función de acompañamiento del aplicador, la necesidad de explicar los ítems, a veces con ejemplos, y su habilidad de, sin dejar de escuchar y tomar nota de sus reflexiones y comentarios, centrarles en lo que estaban haciendo. Todo el material recogido es útil y rico si lo que queremos es definir la forma que tienen de acercarse al conocimiento y aprender. En muchas ocasiones, las cuestiones formuladas les hacen reflexionar sobre ellos mismos, sobre su evolución: «Antes era más impulsivo/a, ahora soy más cobarde». Y otro aspecto no menos importante, la autoestima. Ninguno conocía la existencia y valor de este cuestionario, pero todos se sentían «importantes» haciéndolo.

En el análisis de los resultados destaca la preeminencia de los estilos Reflexivo y Teórico, lo que llevado al terreno de la práctica nos indica que la creatividad queda desbancada en favor de la seguridad. Necesitan saber qué tienen qué hacer, cómo hacerlo y cuáles son los resultados que se esperan. Sería objeto de un estudio amplio y compartido con especialistas el plantear cómo despertar y desarrollar esa creatividad que tanto podría enriquecerles, pero sin que lleguen a perder nunca la seguridad que les puede dar conocer los caminos que deben seguir para alcanzar un objetivo.

Una vez aplicado el cuestionario, detectamos la necesidad de adecuar el contenido y la formulación de los ítems para contextualizarlos a la situación de este tipo de destinatarios, así como la conveniencia de ampliarlo en dos ítems más por estilo, 20 en total, creando así lo que llamamos el CHAEA Sénior que consideramos que puede ser una herramienta que ayude a psicólogos y terapeutas de las residencias de ancianos a diseñar las actividades ocupacionales. 
ENSEÑANZA DEL USO DE LAS TIC A PERSONAS MAYORES:

C. MORENO PABÓN, C. ALCONADA FERNÁNDEZ Y J. DULAC IBERGALLARTU

El método MeTaEducArte trabaja la identidad, las emociones y los sentimientos. Los avances en neurodidáctica nos demuestran que prendemos mejor aquello que sentimos, lo que vivimos y retenemos como un aprendizaje real. Deseamos una educación integral que tenga en cuenta todos los planos de aprendizaje del alumno (corporal, mental y emocional) (Moreno, 20I5, 20I6). Por esto el principal objetivo de MeTaEducArte, además de los objetivos implícitos en el método, es la educación integral, que incluya conocimientos, experimentación y estudio de los cambios de comportamiento emocional, mientras aprendemos (Moreno, 2015, 2016). Nuestros ancianitos quieren aprender de esta manera, no deben ni necesitan rendir cuentas a nadie de lo aprendido, es un autorreconocimiento que eleva su autoestima. La metodología usada en el diseño y desarrollo de estos talleres teórico-práctico está basada en los nuevos métodos de enseñanza, como AAMC, AC y ABP. Estas metodologías han sido utilizadas en conjunción con el método MUPAI (Moreno, 2012, 2013a, 20I3b, 20I4, 20I5; Moreno Pabón y Dulac, 2016).

La investigación Aula Adaptativa promueve una metodología de cambio en las aulas basado fundamentalmente en el uso de las TIC y en otros recursos y herramientas. Este cambio se fundamenta en el desarrollo de la necesaria capacidad para la adaptación, la adecuada formación de los profesores y en la mejor selección de las herramientas y recursos TIC y otros analógicos. www.aulaadaptativa.es.

El tiempo y la coordinación del mismo ha sido un problema a resolver. Ya hemos mencionado que nuestros alumnos necesitaban una atención muy personalizada y que era muy breve el tiempo disponible para hacer una puesta en común o explicación al grupo y pasar posteriormente a una atención de pequeños grupos o individualizada. Los talleres tenían una duración de noventa minutos. Distribuimos los grupos de tres o cuatro alumnos. La dinámica de trabajo que estamos aplicando en las últimas sesiones es aproximadamente la siguiente:

I. Diez minutos de repaso y recordatorio de la última sesión. Utilizamos la pizarra digital con apoyo de imágenes.

2. Diez minutos de motivación inicial y presentación del taller con la temática elegida. Normalmente conmemoraciones: San Isidro, Todos los Santos, Navidad. Presentación en pizarra digital.

3. Veinte minutos de actividad común sobre el taller del día y su temática. Todos participan y quieren ser escuchados. Motivando.

4. Cuarenta minutos para realizar el taller propuesto con diferente herramientas y recursos que iremos rotando por los cuatro o cinco grupos formados. Prestamos mucha atención a la actitud individualizada de algunos participantes para integrarles en el grupo.

5. Diez minutos para recoger y hacer una despedida afectiva. ¿Cuándo vuelven ustedes?, preguntan cariñosamente con interés.

En la misma línea de trabajo que en las aulas con nuestros alumnos, son alumnos investigadores que aprenden investigando, descubriendo y analizando los problemas planteados para aportar soluciones; a nuestros mayores les invitamos a descubrir, investigar, analizar y aprender utilizando el potencial de algunas de las herramientas y recursos TiC que ponemos a su disposición. 


\section{Conclusiones}

Desde los primeros talleres mantenemos la priorización siempre de la motivación emocional y la atención individualizada para integrar un ambiente de aprendizaje adecuado y como garantías de éxito de las sesiones dadas las características de los alumnos.

La mejora notable de la autoestima es uno de los logros que nos resulta más gratificante. Fue un logro que conseguimos desde las primeras sesiones, pero que sigue creciendo en cada uno de los talleres que realizamos.

Las adaptaciones metodológicas deben ser continuas y la diversidad con la que nos encontramos hace que la preparación de los talleres deba adaptarse y que se definirá y concretará en el propio taller. Escuchar y dar respuesta es una necesidad.

En esta fase del proyecto la ratio aconsejada es de un profesor cada cuatro alumnos. Debemos intentar mejorar la autonomía y el acceso a los recursos y herramientas para tratar de bajar esa ratio.

Consideramos muy importante el rápido avance de nuestros mayores en el aprendizaje instrumental de las herramientas y recursos tecnológicos puestos a su disposición por lo creemos que la metodología aplicada es eficaz.

La colaboración del equipo docente con los psicólogos y terapeutas de la residencia ha ido aumentando según avanza el proyecto y es un factor fundamental para su desarrollo, continuidad y mejora en el futuro.

En los próximos meses estamos interesados en detectar mejor los estilos de aprendizaje de las personas mayores basándonos en el aprendizaje con herramientas y recursos Tic.

Vamos a definir la competencia digital básica más adecuada para nuestros mayores. Nos basamos en los intereses y deberemos tener en cuenta la utilización de las herramientas más accesibles para ellos.

Algunas de las mejoras detectadas son las referidas a: atención, colaboración, autoaprendizaje, percepción, memoria, orientación, lenguaje, autoestima, razonamiento, imaginación, emoción, autonomía...

\section{Bibliografía}

Agudo, S.; Pascual, M. A. y Fombona, J. (20I2) Usos de las herramientas digitales entre las personas mayores. Comunicar: Revista Cientifica de Comunicación y Educación, 39, 193-20I.

Alconada, C.; Alonso, C.; Dulac, J. y Gallego, D. (2009) La pizarra digital. Interactividad en el aula. Madrid: Cultiva.

Gallego, D. y Dulac, J. (2005) Informe final del Iberian Research Project. Recuperado de http://www.dulac.es/Iberian\%2oresearch/Informe\% 2ofinal.doc.

Moreno Pabón, C. (20I2) Proyecto de Innovación Docente MeTaEducArte (Método para Talleres de Educación desde el Arte). Nuevos métodos en la educación artística: talleres de arte contemporáneo en la educación primaria e infantil. En M. ${ }^{a}$ V. DE HARO de SAN MATEO, (coord.) Novedades Docentes en el EEES (pp. 7I-96). Madrid (España): Visión libros.

Moreno Pabón, C. (2013) MeTaEducArte (Método para Talleres de Educación desde el Arte). El arte contemporáneo como medio de expresión, en la Educación Infantil y Primaria con uso de Tic. En Estudios sobre el Mensaje Periodístico, vol. 19, núm. especial (marzo 2013) (pp. 339-349). Madrid: Universidad Complutense de Madrid. 
ENSEÑANZA DEL USO DE LAS TIC A PERSONAS MAYORES:

RELATO DE UNA EXPERIENCIA

C. MORENO PABÓN, C. ALCONADA FERNÁNDEZ Y J. DULAC IBERGALLARTU

Moreno Pabón, C. y Dulac, J. (2016) Aula Adaptativa y MeTaEducArte: Proyecto Tic en la tercera edad. En TIC actualizadas para una nueva docencia universitaria (pp. 55I-566). Mc Graw Hill Education.

Pavón, F. y Castellanos, A. (2000) Tecnologías avanzadas: nuevos retos de comunicación para los mayores. Universidad Huelva.

Pujol, R. y Abellán, A. (20I6) Un perfil de las personas mayores en España 20I6. Indicadores estadísticos básicos. Madrid. Informes envejecimiento en Red, n. ${ }^{\circ}$ I4. 
